\title{
Nanoparticles for postinfarct ventricular remodeling
}

\author{
Caijuan Dong ${ }^{1}$, Aiqun Ma*,1 \& Lijun Shang**,2 \\ ${ }^{1}$ Department of Cardiovascular Medicine, First Affiliated Hospital of Xi'an Jiaotong University, Xi'an, Shaanxi, China \\ ${ }^{2}$ School of Chemistry \& Biosciences, University of Bradford, Bradford, BD7 1DP, UK \\ *Author for correspondence: maaiqun@medmail.com.cn \\ **Author for correspondence: I.shang1@bradford.ac.uk
}

In recent years, tremendous progress has been made in the treatment of acute myocardial infarction, but pathological ventricular remodeling often causes survivors to suffer from fatal heart failure. Currently, there is no effective therapy to attenuate ventricular remodeling. Recently, nanoparticle-based drug delivery systems are widely applied in biomedicine especially in cancer and liver fibrosis, owing to its excellent physical, chemical and biological properties. Therefore, the use of nanoparticles as delivery vehicles of small molecules, polypeptides, etc. to improve postinfarct ventricular remodeling is expected. In this review, we summarize the updated researches in this fast-growing area and suggest further works needed.

First draft submitted: 28 July 2018; Accepted for publication: 5 October 2018; Published online: 24 October 2018

Keywords: biomarker $\bullet$ drug delivery $\bullet$ engineering $\bullet$ extracellular vesicles $\bullet$ heart failure $\bullet$ inorganic nanoparticles - liposome • mechanism • myocardial infarction • postinfarct ventricular remodeling

With an aging population and the rising incidence of cardiovascular diseases (CVDs), such as hypertension and coronary heart disease, the prevalence of heart failure is gradually increasing. In 2013, 1 in 9 death certificates (284,388 deaths) in the USA mentioned heart failure, with heart failure being the primary cause in 58,309 of those deaths [1]. In China, the prevalence of heart failure is around $0.9 \%$, and coronary heart disease is the major cause.

Primary myocardial damage (including ischemic myocardial damage like myocardial infarction and immune myocardium damage like myocarditis) and cardiac overload (including pressure overload and volume overload) can impair heart muscle, resulting in compensatory changes such as ventricular hypertrophy or ventricular enlargement, known as 'ventricular remodeling'. In this process, the heart's geometry, cardiomyocytes, interstitial components and the phenotype of cardiomyocytes undergo a series of pathological changes. Ventricular remodeling is the basic mechanism of heart failure and has three main characteristics: pathological cardiomyocyte hypertrophy accompanied by re-expression of embryonic genes, myocytes death, excessive fibrosis or increased degradation of myocardial extracellular matrix (ECM). In recent years, the molecular mechanism of ventricular remodeling has been studied mainly from the following aspects: myocardial hypertrophy, fibrosis, inflammation, mitochondrial dysfunction, autophagy/apoptosis. We may slow the progression of cardiac remodeling by targeting the above mechanisms, thus benefiting heart failure patients.

Nanoparticles refer to particles smaller than $100 \mathrm{~nm}$ in at least one dimension. Due to its small size and excellent thermal and electrical properties, nanoparticles are now widely applied into biomedical and industrial fields such as aerospace, electronics, cosmetics, food additives and optical devices [2]. Meanwhile, considering inherent nanometer size of living cells' biological components, nanotechnology is being considered in various medical fields such as oncology and cardiovascular medicine. Nanoparticles as delivery vehicles of proteins, nucleic acid and small molecules may potentially provide sustained treatment in damaged tissues. Basic research showed that nanoparticles packed with Nox2-NADPH oxidase siRNA, insulin growth factor-1 (IGF-1) or pitavastatin enhanced cardiac function post myocardial infarction (MI) [2-4], suggesting the infinite possibilities of nanoparticles in the treatment of postinfarct ventricular remodeling. 
In this review, we first introduced the present understanding of mechanism of ventricular remodeling and new advances of drugs for ventricular remodeling, then focused on the most commonly used nanoparticles in CVD, especially for postinfarct ventricular remodeling. We also briefly touched on nanoparticles used as diagnostics/biomarkers for CVD, and engineering of nanoparticles for tailored and directed use, which are both growing rapidly in the area.

\section{The mechanism of ventricular remodeling Myocardial hypertrophy}

Myocardial hypertrophy is a powerful adaptive form for various causes of cardiac output reduction, but it is not infinite. If pathological factors last for a long time, the function of the hypertrophic myocardium cannot be maintained persistently and eventually turns to heart failure. At present, plenty of studies support that apelin receptor (APJ receptor), a G protein-coupled receptor, plays a significant part in cardiac hypertrophy [5]. The hypothesis has been raised that APJ internalization via clathrin-mediated endocytic pathway may contribute to myocardial hypertrophy. As the molecular mechanisms of cardiac hypertrophy have been widely studied, the role of long noncoding RNAs has become more prominent [6]. The long noncoding RNA Mhrt (myosin heavy chain-associated RNA transcript) is shown to affect cardiac hypertrophy remodeling by affecting the acetylation of myocardin, a substance which is necessary for programming cardiac muscle [7].

\section{Myocardial fibrosis}

Myocardial fibrosis is known for excessive accumulation of ECM in the myocardium, which is a basic component for most cardiac pathologic changes [8]. Fibrotic cardiac muscle is stiffer and less compliant, causing ventricular systolic and diastolic dysfunction.

\section{Changes in ECM components}

Myocardial ECM is composed of collagen, proteoglycans, glycoproteins, glycosaminoglycans and elastic fibers, which are synthesized and secreted by cardiac fibroblasts. These components are essential for the nutrition and conduction of myocardium, and the normal structure and function of heart. Under the condition of ischemia and hypoxia, cardiac fibroblasts will move to the injured site, and produce a large amount of ECM under the mediation of various cytokines and neurohumoral factors. Epicardium-derived cells, but not bone marrow-derived blood cells, are the main origin of cardiac fibroblasts in the infarcted heart [9]. Significantly, differentiated states of fibroblasts are of importance to postinfarct ventricular remodeling of scarring. Fibroblasts were activated and highly proliferative, reaching a maximum rate within 2-4 days after MI. Up to 3-7 days, these cells differentiated into myofibroblasts and expressed smooth muscle $\alpha$-actin to structurally construct the infarcted zone. As the scar completely formed (around 7-10 days), myofibroblasts gradually lost proliferative ability and smooth muscle $\alpha$-actin expression [10]. This suggests that these fibroblasts might be a target of nanoparticle-mediated drug delivery system (DDS).

Matrix metalloproteinases (MMPs) are a family of zinc-dependent endopeptidases, which can degrade ECM. According to their structure and substrate selectivity, MMPs can be roughly classified into collagenase, gelatinase, matrix lysin, membrane-type metalloproteinase and others. Under normal conditions, MMPs are mostly in the form of zymogens, with low expression levels and weak activity. Once activated under the stimuli of various pathological factors, such as ischemia and hypoxia, MMPs rapidly participate in the degradation of ECM and modulate the signaling pathways related to vascular remodeling and myocardial fibrosis. It has been shown that elevated MMP-9 levels are associated with left ventricular dysfunction and targeted knockout of mouse MMP-9 gene can decrease collagen accumulation post-MI and attenuate left ventricular dilation [11].

\section{Disproportionality of myocardial collagen fiber}

Fibrillar collagen is the major structural protein of cardiac ECM. It is generally classified into Type I (approximately 85\%), Type III (about 11\%) and quite less abundant Types IV, V and VI. Collagen Types I and III have their own functions, that is collagen Type I is responsible for building thick fibers which maintain its tensile strength, while collagen Type III is in charge of constructing thin fibers which keep elasticity of the matrix [12]. Changes in the number, distribution and arrangement of collagen fibers can lead to fibrosis. Cardiac fibroblasts are the major source of collagen fibers in the myocardial infarction. As the most frequently used marker of myocardial fibrosis, collagen peptides can predict cardiac function. For instance, the most common marker of Type I collagen is a $100 \mathrm{kDa}$, C-terminal polypeptide whose plasma levels are positively correlated with diastolic dysfunction; the most 
commonly used marker for the synthesis of Type III collagen is a $42-\mathrm{kDa}, \mathrm{N}$-terminal polypeptide, whose plasma levels are positively associated with mortality and heart failure [13,14].

\section{Inflammation}

Inflammation plays a vital role in ventricular remodeling, and its lasting activation may result in irreversible cardiac damage. Activation of multiple inflammatory factors and signaling pathways have been mentioned in patients with heart failure [15]. Inflammatory molecules involved in ventricular remodeling mainly include TNF- $\alpha$, IL-6, IL-10, IL-18 and nuclear factor- $\kappa \mathrm{B}$. Under the chemotaxis of multiple cytokines, monocytes are recruited to the cardiac tissue from circulation, and then they differentiate into macrophages and contribute to tissue injury and myocardial fibrosis [16]. Infarct macrophages exhibit inflammatory 'M1' phenotype early and reparative 'M2' phenotype later after MI [17]. Numerous studies have shown that the shift from M1 to M2 macrophages can improve postinfarct ventricular remodeling. Future studies can be aimed at targeting specific harmful functions while preserving beneficial effects of macrophages to prevent adverse cardiac remodeling.

NLRP3 inflammasome is a large molecule polyprotein complex, a component of the innate immune system. It is of great importance in both aseptic and infectious inflammation and can be activated by a variety of endogenous and exogenous factors. Studies have revealed that NLRP3 is closely associated with the pathogenesis of heart failure. When the myocardium is in a state of ischemia, NLRP3 is activated, producing IL-1 $\beta$ and IL-18. IL-1 $\beta$ further activates nuclear transcription factor (NF- $\mathrm{B}$ ). In turn, activation of NF- $\mathrm{B}$ can also induce the expression of precursors such as IL-18 and IL-1 $\beta$. IL-18 is associated with the expression of collagen types I and III in a dose-dependent manner and induces myocardial remodeling and interstitial fibrosis.

\section{Mitochondrial dysfunction}

Mitochondrial dysfunction may potentially participate in almost all the mechanisms involved in ventricular remodeling [18]. Varieties of studies have demonstrated that there are functional defects of electron transport chain and oxidative phosphorylation complex in myocardial mitochondria during heart failure. In animal models of heart failure, the activity of myocardial mitochondrial complex IV is significantly reduced and the activity of complexes I and III are inhibited. These changes not only reduce mitochondrial ATP synthesis but also increase mitochondrial reactive oxygen species (ROS) production. ROS modifies the myofibrillar protein of the myocardium through oxidation, resulting in the progressive reduction of cardiac contractility and irreversible cardiac damage [19].

In addition, angiotensin II (Ang II) destroys myocardial mitochondria by increasing the production of ROS and interferes with mitochondrial oxidative phosphorylation, including fatty acid oxidation $[18,20]$. Fatty acids are the main energy substrate of the heart and offer most cofactors essential for mitochondrial oxidative phosphorylation. Studies have shown that overexpression of angiotensinogen in transgenic mice (TG1306/R1 mice) can reduce cardiac fatty acid oxidation and expression of PPAR $\alpha$ protein and fatty acid oxidase [21].

\section{Autophagy \& apoptosis}

Autophagy is the decomposition process of proteins and organelles. It is characterized by the encapsulation of cytoplasmic proteins or organelles to form autophagosomes with a bilayer membrane structure. Autophagosomes fuse with lysosomes to form autolysosomes. The parcels are decomposed into small molecules such as free amino acids and fatty acids and be recycled [22]. Macroautophagy, microautophagy and chaperon-mediated autophagy all allude to autophagy, but here autophagy refers to macroautophagy [23]. Cardiomyocyte autophagy plays a prominent role in maintaining the stability of internal environment, and the structure and function of heart. Ischemia induces autophagy in cardiomyocytes. Kanamori first reported that autophagy was rapidly activated in cardiomyocytes within $30 \mathrm{~min}$ after coronary ligation, and strong autophagic activity was observed particularly in saved cardiomyocytes around the infarcted zone [24]. His following study found that autophagy inhibitor chloroquine can reduce myocardial autophagy and aggravate ventricular dilatation and myocardial remodeling, suggesting that autophagy plays a protective role in postinfarct ventricular remodeling [25]. There are two main pathways for the regulation of myocardial cell autophagy: mTOR-dependent pathway and Beclinl-dependent pathway. Among them, the mTOR-dependent pathway plays a major role in postinfarct ventricular remodeling. mTOR, a serine/threonine protein kinase, is proved to regulate autophagy. Increased mTOR activity inhibits autophagy while decreased mTOR activity activates autophagy [26].

Necrosis has been considered as the only cause of myocyte loss in MI for a long time. However, mounting evidence suggests that apoptosis plays a critical role in the progression of postinfarct ventricular remodeling and 


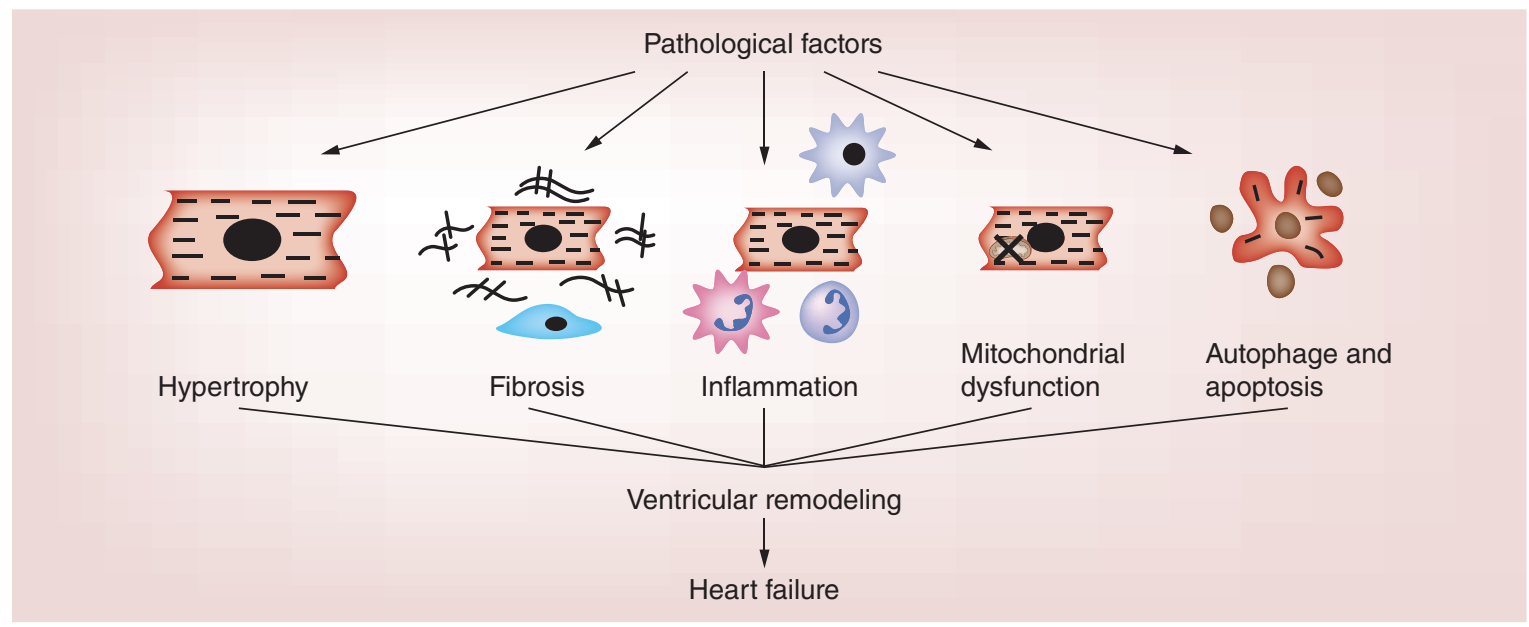

Figure 1. The mechanism of ventricular remodeling. Hypertrophy, fibrosis, inflammation, mitochondrial dysfunction, autophage and apoptosis are involved in the pathways of ventricular remodeling.

\begin{tabular}{|c|c|c|c|c|}
\hline Classification & Typical Drugs & Function & Study & Ref. \\
\hline RAAS inhibitors & $\begin{array}{l}\text { Aliskiren, aldosterone, } \\
\text { eplerenone }\end{array}$ & $\begin{array}{l}\text { Inhibit cardiac hypertrophy and reduce the } \\
\text { proliferation of extracellular matrix and } \\
\text { interstitial fibrosis }\end{array}$ & $\begin{array}{l}\text { Gheorghiade et al. } \\
\text { Dhillon et al. }\end{array}$ & {$[32]$} \\
\hline$\beta$-Adrenoceptor blockers & $\begin{array}{l}\text { Atenolol, metoprolol tartrate } \\
\text { tablets, propranolol }\end{array}$ & $\begin{array}{l}\text { Block the sympathetic nervous system, RAAS } \\
\text { system and reduce oxygen consumption of heart } \\
\text { muscle }\end{array}$ & Xie et al. & [34] \\
\hline HMG-COA inhibitors & Atorvastatin rosuvastatin & $\begin{array}{l}\text { Inhibit oxidation and inflammation, reduce } \\
\text { sympathetic nerve activity and improve vascular } \\
\text { endothelial function }\end{array}$ & $\begin{array}{l}\text { Duan et al. } \\
\text { Mahalwar et al. }\end{array}$ & $\begin{array}{l}{[35]} \\
{[36]}\end{array}$ \\
\hline $\begin{array}{l}\text { Vascular endothelin-1 (ET-1) } \\
\text { Receptor antagonists }\end{array}$ & $\begin{array}{l}\text { Bosenta, enrasentan, } \\
\text { tezosent, sitaxentan, } \\
\text { atrasentan, ambrisentan }\end{array}$ & $\begin{array}{l}\text { Contract blood vessels, promote cell mitosis and } \\
\text { proliferation. }\end{array}$ & Prasad et al. & [37] \\
\hline New vasodilators & Sildenafil, AVE9488 & Inhibit cardiac hypertrophy, apoptosis and fibrosis & $\begin{array}{l}\text { Vecchis et al. } \\
\text { Fraccarollo et al. }\end{array}$ & $\begin{array}{l}{[38]} \\
{[39]}\end{array}$ \\
\hline
\end{tabular}

heart failure, too. Cardiac-specific caspase-3 is the most important caspase in the terminal apoptotic pathway in the infarcted heart. The study found that overexpression of caspase- 3 increased infarct size in transgenic mice [27]. Vice versa, reduced caspase- 3 narrowed the infarct size, lowered the number of apoptotic cardiomyocytes and promoted the cardiac function in MI mice [28,29]. One study from Zhou et al. found that isoproterenol induces abnormal endoplasmic reticulum stress through inactivation of AMPK, which in turn damages cardiomyocytes, causes apoptosis and leads to heart failure in rats, and all the processes can be suppressed by activating AMPK, providing a potential mechanism of apoptosis [30].

In conclusion, none of the above processes is independent. On the contrary, they interact with each other and form a complex mechanism network. Nanoparticles loaded with the drugs targeting any of these mechanisms are expected to reverse ventricular remodeling and prevent further progression of the disease. We summarized the mechanism of ventricular remodeling in Figure 1.

\section{Nanocargo for ventricular remodeling}

Drugs, proteins, RNA and other small molecules all can be the cargo of nanoparticle-based DDS, which we describe below in details.

\section{Drugs}

We summarized new advances of drugs for ventricular remodeling in Table 1. The recent development of cardiovascular medicine and coronary intervention like PCI greatly decreased the mortality of acute myocardial infarction 


\begin{tabular}{|c|c|c|c|}
\hline Proteins & Cardioprotective effect & Study & Ref. \\
\hline VEGF & Promote cardiac stem cells differentiation into vascular endothelial cells & Xiao et al. & [41] \\
\hline IGF-1 & Promote stem cell growth and differentiation & Jackson et al. & [42] \\
\hline Thymosin $\beta 4$ & Promote neoangiogenesis and cardiac regeneration & $\begin{array}{l}\text { Srivastava et al. } \\
\text { Shrivastava et al. }\end{array}$ & $\begin{array}{l}{[43]} \\
{[44]}\end{array}$ \\
\hline FAP- $\alpha$ & Promote fibroblast migration & Tillmanns et al. & [45] \\
\hline HIF- $1 \alpha$ & $\begin{array}{l}\text { Promote cardiac function, angiogenesis, cardiomyocyte proliferation and reduction of } \\
\text { fibrotic tissue with no induction of cardiac hypertrophy }\end{array}$ & Cerrada et al. & [46] \\
\hline Wnt $1 / \beta$ catenin & Induces cardiac fibroblasts to proliferate and express pro-fibrotic genes & Duan et al. & [47] \\
\hline TGF- $\beta$ & $\begin{array}{l}\text { Activate a pro-fibrotic and matrix-preserving program in infarct fibroblasts through } \\
\text { Smad-dependent actions }\end{array}$ & Shinde et al. & [48] \\
\hline
\end{tabular}

FAP- $\alpha$ : Fibroblast activation protein $\alpha$; HIF-1 $\alpha$ : Hypoxia-inducible factor-1 $\alpha$; IGF-1: Insulin-like growth factor-1; TGF- $\beta$ : Transforming growth factor $\beta$; VEGF: Vascular endothelial growth factor.

(AMI), but the incidence of chronic heart failure patient's post-MI is increasing. Nanotechnology-based DDS (nano-DDS) is a hot topic in drug delivery and has been successfully adopted in cancer therapy. Besides higher biocompatibility, nano-DDS can increase the precision of drug targeting and the level of drug accumulation in the desired area, which will be discussed in detail in later sections. Therefore, nano-DDS has great potential in CVDs. As is known to us all, statins have anti-inflammatory effects. Katsuki et al. utilized PLGA as the carrier of Pitavastatin and tested its efficacy in MI model. They found intravenous treatment with PLGA-Pitavastatin nanoparticles attenuated postinfarct ventricular remodeling by interfering with monocyte recruitment and reducing monocyte/macrophage accumulation in the heart. This indicated that inflammatory cells might be the target of PLGA-Pitavastatin nanoparticles [31].

However, not all the drugs could be nanoparticulated in view of their size and structure. Furthermore, there are few animal studies using nanoparticles in the treatment of heart diseases, albeit clinical trials. For example, Zhang et al. showed size-dependent cardiac effects of gold nanoparticles (AuNPs) in isoproterenol-induced hyperthyroid rats, suggesting that size is an important factor affecting safety and efficacy of nano-DDS [40]. Further extensive work is needed to explore the target cells and examine the efficacy and safety of these cardioprotective drugs with nano-DDS before going into clinical trials.

\section{Proteins}

We summarized some proteins which contribute to cardiac repair and attenuate adverse remodeling in Table 2. Under ischemic conditions, certain proteins can play important roles in attenuating and delaying pathological ventricular remodeling. However, their effect is quite limited as a result of short half-lives in vivo in their free form. What if they are nanoparticulated? In fact, some of them have been nanoparticulated for use in the treatment of postinfarct ventricular remodeling in animal experiments. For example, injecting PLGA-IGF-1 nanoparticles into the myocardium of MI mice was effective to narrow infarct size, prevent cardiomyocyte apoptosis and improve left ventricle ejection fraction 3 weeks after the left coronary ligation surgery [3]. But similar animal studies are still lacking, and transition from basic research into clinical trials is near to zero. Future studies should focus on this part of works and provide more reliable data.

\section{Ribonucleic acid}

miRNAs are small, noncoding, single-stranded RNAs, which can regulate the gene expression by interacting with target mRNAs and preventing them translating into functional proteins. It has been shown that miR-1, miR-21, miR145, etc. modulated the gene expression relative to ventricular remodeling. If these miRNAs are nanoparticulted and delivered to the infarcted heart, it may prevent adverse ventricular remodeling. In fact, extracellular vesicles (EVs) are natural delivery vehicles of miRNA and have been used in the treatment of ventricular remodeling in basic research, which will be discussed in detail in later part. Other kinds of nanoparticles such as liposomes are also studied. For example, one study investigated that miR-145 encapsulated in liposomes was intravenously administered to MI rabbit and had a cardioprotective effect by inducing cardiomyocyte autophagy through targeting fibroblast growth factor receptor substrate 2 [49]. 
Small hairpin RNA, an artificial RNA, is named for a tight hairpin turn that can be used to silence specific gene expression through RNA interference and reduce the level of targeted proteins. As an example, one study exhibited that $\mathrm{rPEI} / \mathrm{siRAGE}$ showed high target gene silencing and low toxicity in cardiomyocytes, and the treatment of $\mathrm{rPEI} /$ siRAGE reduced the myocardial infarction size [50].

\section{Nanoparticles for treatment of postinfarct ventricular remodeling}

Heart failure post-MI is an important component of cardiovascular related diseases. As mentioned before, the repair mechanism of post-MI causes collagen scarring to replace damaged cardiomyocytes. The nonshrinkage of the scar leads to viable cardiac hypertrophy, thinning of the infarct wall and expansion of the ventricles, which is known as ventricular remodeling. Without effective treatment, these processes will continue until the heart no longer adequately pumps enough blood to the body, which is defined as heart failure. At present, the drugs used for the treatment of heart failure only aim at its pathophysiological process, but do not target the cause. Worse still, the current available means of delivering cardioprotective drugs often misses the deadline of reversible repair of cardiomyocytes and inevitably, cardiomyocytes undergo a series of remodeling processes. Therefore, developing novel therapies to alleviate the negative left ventricular remodeling post-MI is very important.

Nanoparticles refer to nanometer-sized particles. It is defined as particles smaller than $100 \mathrm{~nm}$ in at least one dimension. Due to its small size and excellent thermal and electrical properties, nanoparticles are now widely used in industrial and biomedical fields such as cosmetics, food additives, aerospace, electronics and optical devices [2]. Considering the intrinsic nanometer size of the living cell's biological components, if nanoparticles are designed as delivery vehicles of a variety of growth factors, enzymes and small molecule, the modified nanoparticles may provide infinite possibilities in the treatment of CVDs. In addition, nanoparticles can achieve controlled drug release by constructing a release system in response of internal stimuli such as $\mathrm{pH}$, redox state and the presence of biomolecules as well as external stimuli such as light and magnetic field. One study showed that $\mathrm{pH}$-sensitive mesoporous silica-based nanoparticles could be mostly taken up by tumor cells for their acidic environment due to hypoxia [51]. Similarly, an infarcted heart is also in an acidic environment under ischemic condition. pH-sensitive nanoparticles can also be designed to achieve controlled drug release. In addition, if the nanoparticles can only be opened by specific enzyme which is limited to infarct heart, the controlled drug release can also be achieved. Currently, the most commonly used nanoparticles in cardiovascular research are inorganic nanoparticles, liposomes and EVs, which are discussed below.

\section{Inorganic nanoparticles}

Common inorganic nanoparticles include AuNPs, silver nanoparticles and silicon nanoparticles and so on. They are widely studied in basic research associated with CVDs.

\section{Gold nanoparticles}

AuNPs have great potential in the diagnosis and treatment of various diseases, owing to their superior physicochemical and pharmacological characters. In terms of physicochemical properties, AuNPs are inert, stable and biocompatible, which means low toxicity. Moreover, the surface of AuNPs can be retouched as we wish, to improve precision and safety of its application in clinical or research [52]. As for the preparation process, AuNPs are easy to prepare and functionalize, which may lower the production cost, and this obvious economic advantage will make them more available in clinical. Based on the above, AuNPs are a valuable candidate for many biomedical applications, such as drug delivery, cancer therapy and biomedical imaging. This part focuses on the previous studies of AuNPs in drug delivery. One study showed that continuous injection of gold nanofibers combined with platelet-derived growth factor into the heart of rats could reduce myocardial cell death and maintain systolic function post-MI. Considering that heart failure is related to abnormal electrical function, modified nanofibers to couple the electrical property was studied. The result showed that ex vivo pretreatment of mesenchymal stem cells (MSCs) using 5-azacytidine and AuNPs loaded with conductive nanofibrous construct could improve cardiomyogenic differentiation, thus resulting in protective effects on infarcted area [53]. Another study showed that PEG-coated AuNPs could attenuate $\beta$-adrenergic receptor-mediated acute cardiac hypertrophy and inflammation by inhibiting the expression of $\beta 1-\mathrm{AR}$ and its downstream effectors IL-6 and ERK1/2 [52]. In conclusion, AuNPs have at least two functions for treating heart failure: one is used as drug vector and the other is used as anti-cardiac hypertrophy agents. 


\section{Silicon nanoparticles}

Silicon nanoparticles are also drawing more and more attention. Porous silicon (PSi) shares the similar characters with AuNPs such as nanoscale size, large surface area $\left(>300 \mathrm{~m}^{2} / \mathrm{g}\right)$ and good biocompatibility and biodegradability [54,55]. Besides, high degree of porosity (50-80\%) and the unique chemical surface are two special features of PSi, which can promote the solubility of hydrophobic drugs and control the drug release. An in vivo experiment tested the biocompatibility of different sizes of thermally oxidized PSi nanoparticles in the heart tissue. Obvious changes in cardiac function and other systems like the hematological system were not found before and after MI, demonstrating that thermally oxidized PSi nanoparticles have good biocompatibility [56]. Other research concluded that functionalized undecylenic acid thermally carbonized PSi nanoparticles can improve their ability of accumulation in different cardiomyocytes (primary cardiomyocytes, noncardiomyocytes and H9C2 myocardium). It has also been reported that intravenous administration of peptide-modified nanoparticles in MI rats induced by isoproterenol, could increase the accumulation of nanoparticles in the heart up to 3.0 times in $10 \mathrm{~min}$, revealing the potential therapeutic effect of these peptide-modified nanosystems in postinfarct ventricular remodeling. Therefore, we can confidently expect that the PSi materials modified with heart-targeting peptides and cardioprotective factors might serve as a promising approach to treating heart diseases.

Nanoparticles mentioned above are delivered by directly injecting into the infarcted heart or through the intravenous administration. Some studies also deliver nanoparticles orally. However, this route of administration can cause an undesirable systemic response for their inherent metabolic pathways. Currently, drug delivery using inhaled nanoparticles has demonstrated a promising future [57-59]. For example, inhalation of calcium phosphate nanoparticles bound to peptides targeting heart were investigated and no changes in blood pressure, heart rate or respiratory function were observed [58]. Therefore, efforts to develop tailored approaches with engineering designs are a hot topic in the study, as described in part 'engineering precison nanoparticles for personalized CVDs'.

It is worth noting that inorganic nanoparticles can also cause toxic effects in multiple organs. Experiments conducted on murine macrophage cell lines have shown that AuNPs could affect the immune system. In this study, AuNPs successfully reduced activation of Toll-like receptors-9 receptors, which in turn reduced the secretion of various interleukins (IL-6, IL-12) and TNF- $\alpha$ [60]. Silica nanoparticles can cause toxic effects, too. The elevated level of pro-inflammatory cytokines like IL-6 and IL-12, increased inflammatory cells such as natural killer cells and cytotoxic T-cells and upregulated genes associated with inflammatory responses were observed when cells were exposed to silica nanoparticles [61]. Besides, the level of cardiac muscle troponin (cTnT), a sensitive indicator of AMI, was significantly elevated in old rats exposed to aerosol of silica nanoparticles [62], which exhibited cardiotoxicity.

\section{Liposomes}

Liposomes are composed of single or multiple outer lipid bilayers and an inner aqueous compartment, thus endowed with special properties that can be combined with both hydrophobic and hydrophilic materials. The diameter of liposomes is usually $20-10 \mu \mathrm{m}$, and the thickness of phospholipid bilayer is around $4-5 \mathrm{~nm}$. Microfluidic technology has gradually been employed for liposome preparation in place of conventional methods [63-65]. At present, PLA, PGA and PLGA are three popular materials for the preparation of liposomes. Initially, liposomes were used in surgical implants and tissue repair. They were then rapidly introduced into other medical fields such as abdominal mesh and drug-delivery vectors due to their excellent biocompatible, biodegradable and nontoxic properties [66-68]. Another unique advantage of using PLA to make nanoparticles is flexibility. Its physical properties as well as chemical properties are easily changed to obtain the desired pharmacokinetics and biodegradable properties $[68,69]$. Nanoliposomes have been shown to transport many substances such as low molecular weight drugs, imaging agents, peptides, proteins and nucleic acids. They can slowly release the encapsulated drug, maintain a higher drug concentration in the target area, and increase drug efficacy. This ability can also be applied to delivery of cardioprotective agents to the infarcted heart.

\section{Ribonucleic acid}

Nox2-NADPH, the major source of ROS in the heart, is associated with ventricular remodeling. It has been reported that the expression of Nox2-NADPH is upregulated in the infarcted myocardium and deleting the gene of Nox2 can reduce oxidative stress and prevent adverse postinfarct remodeling. Considered above, a study used acid-degradable polyketal particles as delivery vehicles for Nox2-siRNA and found that intramyocardial injection of Nox2-siRNA nanoparticles to the post-MI heart successfully prevented upregulation of Nox2 and dramatically improved cardiac function [2]. 


\section{Proteins \& peptides}

A study reported that the amount of angiotensin II Type 1 (AT1) nanoliposomes clustered in the infarcted heart after day 1 accounted for almost half of the total amount accumulated in all organs, exhibiting superior cardiactargeted capability [70]. IGF-1, a kind of peptide, has been revealed to improving cardiomyocyte growth and survival both in vitro and in vivo. An animal experiment showed that injecting PLGA-IGF-1 nanoparticles (NPs) into the myocardium of MI mice was effective in narrowing infarct size, preventing cardiomyocyte apoptosis and improving left ventricle ejection fraction 3 weeks after the surgery [3].

Drugs

It is well known that inflammatory responses play a critical role in the development of postinfarct remodeling [71]. For this reason, a study explored the effects of PLGA NPs combined with the anti-inflammatory drug-pitavastatin on post-MI mice and concluded that treatment with PLGA-pitavastatin NPs alleviated postinfarct remodeling along with a reduction of monocytes/macrophages in the heart, yet the control group did not. Specifically, PLGApitavastatin NPs not only prevented monocytes from mobilizing in the spleen in MI mice, but also decreased the amount of inflammatory cells in the infarcted heart in splenectomy mice 4. This suggests that nanoparticles loaded with pitavastatin or other similar drugs may be a novel therapeutic strategy to protect the heart from inflammatory damage.

Compared with inorganic nanoparticles, there are few reports about the toxicity of liposomes. Lutein-PLGA nanoparticles were prepared and tested for acute, subacute toxicity, bioavailability and tissue distribution in mice. The results did not show any significant changes in mortality, hematology and other vital organs compared with the control group [72]. However, long-term toxicity studies are needed.

\section{Extracellular vesicles}

EVs, which are of nanoscale size and surrounded by a phospholipid bilayer, are secreted by almost all cell types into extracellular space. EVs are originally considered as cellular debris, but more value in carrying and exchanging biological information is explored with the in-depth research. According to the vesicles' size, biogenesis and surface markers, EVs can generally be divided into exosomes, microvesicles/ectosomes and apoptotic bodies. Exosomes, roughly 30-100 nm in size, are derived from late endosomes. Microvesicles, ranging between 100 and $1000 \mathrm{~nm}$, are generated by budding from the plasma membrane. Apoptotic bodies are derived from apoptotic cells and are more than $1000 \mathrm{~nm}$ in diameter. However, due to the different methods of isolating and purifying vesicles, it is difficult to classify EV precisely. Clinical applications of EVs fall into two main categories: first, EVs are used as biological drugs. Some of the molecules contained in EVs secreted by specific cells have therapeutic effects such as immunosuppression, immune activation, tissue repair and cardiac protection [73]. Second, EVs are used as the drug-delivery system. EVs can serve as a natural nano-DDS to deliver drugs to target cells. Currently, EVs have been used successfully as a carrier for the delivery of small molecules, siRNAs, proteins and mRNAs for preclinical studies.

One study surprisingly found that although there are many kinds of cells in the heart tissue such as cardiomyocytes, endothelial cells and fibroblasts, $\mathrm{EV}$ s from $\mathrm{CD} 34^{+}$stem cells were only selectively captured by cardiomyocytes and endothelial cells, indicating the existence of cell-specific receptors on EVs from CD34 ${ }^{+}$stem cells [74]. Increasing evidence has confirmed that EVs are enriched in miRNAs, which regulate growth, proliferation and survival. More interestingly, miRNAs enclosed in different EVs are quite different from each other and perform different tasks. It has been demonstrated that cardiac progenitor cell-derived exosomal particles which contain miR-21 could prevent cardiomyocytes apoptosis [75]. Other similar studies investigated that miR-132 containing exosomes derived from pericyte had a pro-angiogenic capacity and miR-155 containing exosomes could mediate macrophage-fibroblast interactions and protect damaged myocytes by inhibiting fibroblast proliferation [76]. All of these highlight that understanding how EVs are released and taken up would help to manage and govern postinfarct ventricular remodeling. Future studies might aim at targeted delivery of EVs containing beneficial factors to heart. We summarized the latest findings of exosomes and their miRNAs on cardiac repair in Table 3.

Compared with liposomes, EVs have the following characters: EVs contain a lipid layer and have a better fluidity; membrane proteins contained in the lipid layer of EVs are potentially targeted; EVs can be modified to carry the required membrane fusion and cell-uptake devices; EVs are similar to the body's own cells, so they are non-immunogenic and biocompatible; and EVs are self-cell products which can avoid being degraded by macrophages, endosomal and lysosomal pathways. These natural features make EVs easier to be captured by target 
Table 3. Exosomes and the miRNAs on cardiac repair.

\begin{tabular}{|c|c|c|c|c|c|}
\hline Cellular origins of exosomes & Contained miRNAs & Mechanism & Cardioprotective function & Study & Ref. \\
\hline Cardiac-derived progenitor cells & miR-1 & $\begin{array}{l}\text { Target and inhibit the expression of } \\
\text { sprouty-related EVH1 } \\
\text { domain-containing protein } 1 \text { (Spred1) }\end{array}$ & $\begin{array}{l}\text { Enhance angiogenesis and } \\
\text { potentially improve } \\
\text { cardiac regeneration }\end{array}$ & van Mil et al. & [77] \\
\hline $\begin{array}{l}\text { Induced pluripotent stem cells } \\
\text { (iPS cells), derived from somatic } \\
\text { cells reprogrammed by four stem } \\
\text { cell transcription factors, Oct4, } \\
\text { Sox2, KIf4 and c-Myc }\end{array}$ & miR-21, miR-210 & $\begin{array}{l}\text { Associate with Nanog, an embryonic } \\
\text { stem cell-specific transcription factor } \\
\text { and the key hypoxia related } \\
\text { transcription factor HIF- } 1 \alpha .\end{array}$ & $\begin{array}{l}\text { Reduce oxidative stress } \\
\text { and promote } \\
\text { cardiomyocytes survival }\end{array}$ & Wang et al. & [78] \\
\hline MSC overexpressing GATA-4 & miR-19a, miR-451 & $\begin{array}{l}\text { Activate the Akt and ERK signaling } \\
\text { pathways }\end{array}$ & Reduce apoptosis & Yu et al. & [79] \\
\hline $\begin{array}{l}\text { Human } \mathrm{CD} 34^{+} \text {peripheral } \\
\text { blood-derived hematopoietic } \\
\text { stem cells }\end{array}$ & miR-126, miR-130a & $\begin{array}{l}\text { Increase production of } \mathrm{CXCL} 12 \text { in } \\
\text { endothelial cells and reduce protein } \\
\text { levels of phosphoinositol-3 kinase } \\
\text { regulatory subunit } 2 \text { (PIK3R2) }\end{array}$ & Promote angiogenesis & Mocharla et al. & [80] \\
\hline MSCs & $\operatorname{miR}-22$ & $\begin{array}{l}\text { Target methyl CpG binding protein } 2 \\
\text { (Mecp2) }\end{array}$ & $\begin{array}{l}\text { Reduce apoptosis and } \\
\text { cardiac fibrosis }\end{array}$ & Feng et al. & [81] \\
\hline SVP progenitor cells & $\operatorname{miR}-132$ & $\begin{array}{l}\text { Inhibit Ras-GTPase activating protein } \\
\text { and methyl-CpG-binding protein } 2\end{array}$ & $\begin{array}{l}\text { Improve contractility, } \\
\text { reparative angiogenesis } \\
\text { and interstitial fibrosis }\end{array}$ & Katare et al. & [82] \\
\hline
\end{tabular}

cells and enhance drug delivery efficiency, opening up new ways for drug delivery. However, there is still a long way to fully master physiological characteristics of EVs and improve EVs isolation and drug loading techniques, finally realizing the changes from basic to clinical practice.

\section{Nanoparticles used as diagnostics \& biomarker for CVD}

Nanoparticles are increasingly being recognized as markers of disease presence and prognosis like cancer and CVDs [83,84]. Previously noted, EVs are produced by budding from cytoplasm. Therefore, their contents indirectly reflect the type and condition of original cells. By detecting EVs in the serum, urine and other bodily fluids, we may identify the disease presence and evaluate prognosis. For instance, hypoxia and oxidative stress altered the proteomic and RNA content of endothelial and mast cells [85]. Increased microvesicle levels were found in acute coronary syndrome than that in stable angina [84]. Some clinical research suggested that plasma levels of exosomes subtypes might be used as indicators of vascular endothelium damage in CVD, but more work needed in exploring the potential and attractive ability of EVs.

\section{Engineering precision nanoparticles for personalized CVDs}

Current efforts in nanoparticle work develop increasingly toward tailored approaches and for this purpose nanoparticles, including EV therapy for heart disease, are being modified more and more, such as enzyme prodrug therapy and microfluidic technology. Enzyme prodrug therapy makes it possible to release drugs in desired positions by converting inactive prodrugs to active drugs with the participation of enzymes [86]. Microfluidic extrusion approaches for fabricating exosome mimetics from donor cells and microfluidic surface engineering of living-cell-derived exosomes are two research direction in microfluidic technology, but still face lots of challenges [87]. Magnetically sensitive microcapsules have provided a novel method of targeted cell delivery. Magnetically sensitive cells which contain magnetic nanoparticles in their walls can transfer to the desired sites and repair the damaged tissue in applied magnetic field [88]. However, there is almost no research on the application of magnetic sensitive microcapsules to CVDs.

\section{Conclusion}

CVD is one of the leading causes for death. Although great advances in therapy strategies have been achieved in AMI in recent years, pathological ventricular remodeling often causes survivors to suffer from fatal heart failure. Nanoparticles as emerging drug-delivery vehicles are drawing more and more attention, especially in cardiovascular areas. In this review, we summarized current knowledge about nanomedicine for postinfarct ventricular remodeling, and described basic mechanisms of ventricular remodeling, including hypertrophy, fibrosis, inflammation, mitochondrial dysfunction, autophagy and apoptosis. A better understanding of the mechanisms would help us to find 
new therapeutic targets for ventricular remodeling. We also listed potential nanocargo such as drugs, proteins and RNA for postinfarct ventricular remodeling. Once nanoparticulated, these drugs, proteins and RNA might have better cardioprotective effects. Furthermore, we summarized the characteristics of inorganic nanoparticles, liposomes and EVs and listed their application in postinfarct ventricular remodeling. Nanoparticle-based DDSs show high compatibility, low toxicity, high drug accumulation and controllable drug release. To broaden the knowledge of nanomedicine, we also briefly described the engineering of nanoparticles.

\section{Future perspective}

To date, nanoparticles packed with peptides, siRNA and other small molecules are observed to exert beneficial effects on damaged cardiac myocytes and the infarcted heart both in vitro and in vivo. What is more, nanoparticles are shown to be potential biomarkers of CVD. Nonetheless, there are still some problems in the application of nanoparticles for treating postinfarct ventricular remodeling. First, nanoparticles lack specific markers for targeting cardiac myocytes. It can cause drug accumulation in other relevant target organs and correspondingly produce toxic and side effects. Second, the study of nanoparticles for improving cardiac remodeling is lacking the combination of long-term and short-term animal experiments. The commonly accepted method for establishing the model of AMI in rats is left anterior descending artery ligation. The surgery is very demanding on the operator's skill and is extremely traumatic for rats. Therefore, the long-term survival rate of rats (more than 3 weeks) is quite low. The future work should focus on the long-term animal experiments and investigate the long-term effects of nanoparticles to the infarcted animals. Third, there are few studies on the effects of nanoparticles on ion channels in cardiac myocytes. The ion channel is a popular and challenging topic in cardiac research. Do nanoparticles affect the ion channels in cardiac myocytes? If yes, what is the mechanism? This may be a direction for future research on nanoparticles. Fourth, there is still great technical difficulty and cost concerns in the manufacturing of ideal combination of drugs and nanoparticles. The nanoparticles need to be well engineered to achieve desired size, shape, surface charge and other physicochemical properties, thus a complex and costly method is demanded. Incomplete characterization of nanoparticles can lead to various biological effects. In addition, materials with combination of less toxicity and side effects, better biocompatibility and more controllable drug release will pose major challenges to the materials industry. Fifth, nanoparticles for the treatment of postinfarct ventricular remodeling are still lacking clinical data. Current research in this area is still limited to animal and cell experiments and there are no reliable clinical trials to support the idea. More work is urgently needed to achieve clinical transition of nanoparticles. 3D printing has gained a significant focus in biomedical and nanobiomaterials research. Future studies may adopt 3D printing technology in nanoparticle-based DDSs.

On a positive note, nanoparticles as the delivery vehicle of various drugs have already entered clinical research for the treatment of hepatic fibrosis and cancer. This brings hope to nanoparticles for postinfarct ventricular remodeling.

\section{Executive summary}

- The prevalence of heart failure is gradually increasing and ventricular remodeling is the major cause.

- Nanoparticles have great potential as drug delivery vehicles in postinfarct ventricular remodeling.

The mechanism of ventricular remodeling

- Hypertrophy, fibrosis, inflammation, mitochondrial dysfunction, autophagy and apoptosis are the basic mechanisms of ventricular remodeling.

Nanocargo for ventricular remodeling

- Nanoparticulation of drugs, proteins and RNA is expected to treating postinfarct ventricular remodeling.

Nanoparticles for treatment of postinfarct ventricular remodeling

- Inorganic nanoparticles, liposomes and extracellular vesicles as delivery vehicles of RNA, protein, drugs, and so forth, have been studied in basic research in the treatment of postinfarct ventricular remodeling.

Nanoparticles used as diagnostics \& biomarker for cardiovascular diseases

- Nanoparticles are increasingly being recognized as markers of presence and prognosis of cardiovascular diseases.

Engineering precision nanoparticles for personalized cardiovascular diseases

- Enzyme prodrug therapy and microfluidic technology increase the precision of targeting. 
Financial \& competing interests disclosure

The authors have no relevant affiliations or financial involvement with any organization or entity with a financial interest in or financial conflict with the subject matter or materials discussed in the manuscript. This includes employment, consultancies, honoraria, stock ownership or options, expert testimony, grants or patents received or pending, or royalties.

No writing assistance was utilized in the production of this manuscript.

\section{Open access}

This work is licensed under the Attribution-NonCommercial-NoDerivatives 4.0 Unported License. To view a copy of this license, visit http://creativecommons.org/licenses/by-nc-nd/4.0/

\section{References}

Papers of special note have been highlighted as: • of interest; $\bullet \bullet$ of considerable interest

1. Mozaffarian D, Benjamin EJ, Go AS et al. Executive summary: heart disease and stroke statistics—2016 update. Circulation 133(4), 447-454 (2016).

2. Somasuntharam I, Boopathy AV, Khan RS et al. Delivery of Nox2-NADPH oxidase siRNA with polyketal nanoparticles for improving cardiac function following myocardial infarction. Biomaterials 34(31), 7790-7798 (2013).

- Details how to deliver Nox2-NADPH oxidase siRNA with polyketal nanoparticles in postinfarct ventricular remodeling.

3. Chang M, Yang Y, Chang C et al. Functionalized nanoparticles provide early cardioprotection after acute myocardial infarction. $J$. Control. Release 170(2), 287-294 (2013).

- Explains how nanoparticles can be protective after acute myocardial infarction.

4. Mao Y, Koga JI, Tokutome M et al. Nanoparticle-mediated delivery of pitavastatin to monocytes/macrophages inhibits left ventricular remodeling after acute myocardial infarction by inhibiting monocyte-mediated inflammation. Int. Heart J. 58(4), 615-623 (2017).

-. Updated study about nanoparticles used in postinfarct ventricular remodeling and includes animal experiments.

5. He L, Chen L, Li L. The mechanosensitive APJ internalization via clathrin-mediated endocytosis: a new molecular mechanism of cardiac hypertrophy. Med. Hypotheses 90, 6-10 (2016).

6. Luo Y, Xu Y, Liang C, Xing W, Zhang T. The mechanism of myocardial hypertrophy regulated by the interaction between Mhrt and myocardin. Cell Signal. 43, 11-20 (2018).

7. Han P, Li W, Lin CH et al. A long noncoding RNA protects the heart from pathological hypertrophy. Nature 514(7520), 102-106 (2014).

8. Kong P, Christia P, Frangogiannis NG. The pathogenesis of cardiac fibrosis. Cell. Mol. Life Sci. 71(4), 549-574 (2014).

9. Ruiz-Villalba A, Simón AM, Pogontke C et al. Interacting resident epicardium-derived fibroblasts and recruited bone marrow cells form myocardial infarction scar. J. Am. Coll. Cardiol. 65(19), 2057-2066 (2015).

10. Fu X, Khalil H, Kanisicak $\mathrm{O}$ et al. Specialized fibroblast differentiated states underlie scar formation in the infarcted mouse heart. J. Clin. Invest. 128(5), 2127-2143 (2018).

11. Yabluchanskiy A, Ma Y, Iyer RP, Hall ME, Lindsey ML. Matrix metalloproteinase-9: many shades of function in cardiovascular disease. Physiology (Bethesda) 28(6), 391-403 (2013).

12. Fan D, Takawale A, Lee J, Kassiri Z. Cardiac fibroblasts, fibrosis and extracellular matrix remodeling in heart disease. Fibrogenesis Tissue Repair 5(1), 15 (2012).

13. Cicoira M, Rossi A, Bonapace S et al. Independent and additional prognostic value of aminoterminal propeptide of type III procollagen circulating levels in patients with chronic heart failure. J. Card. Fail. 10(5), 403-411 (2004).

14. Ma Y, de Castro BL, Toba $\mathrm{H}$ et al. Myofibroblasts and the extracellular matrix network in post-myocardial infarction cardiac remodeling. Pflugers Arch. 466(6), 1113-1127 (2014).

15. Ong SB, Hernandez-Resendiz S, Crespo-Avilan GE et al. Inflammation following acute myocardial infarction: multiple players, dynamic roles, and novel therapeutic opportunities. Pharmacol. Ther. 186, 73-87 (2018).

16. Passino C, Barison A, Vergaro G et al. Markers of fibrosis, inflammation, and remodeling pathways in heart failure. Clin. Chim. Acta 443, 29-38 (2015).

17. Ma Y, Mouton AJ, Lindsey ML. Cardiac macrophage biology in the steady-state heart, the aging heart, and following myocardial infarction. Transl. Res. 191, 15-28 (2018).

18. Schirone L, Forte M, Palmerio $S$ et al. A review of the molecular mechanisms underlying the development and progression of cardiac remodeling. Oxid. Med. Cell. Longev. 2017, 1-16 (2017).

- Summarizes the basic and updated molecular mechanisms of pathological cardiac remodeling.

19. Rosca MG, Vazquez EJ, Kerner J et al. Cardiac mitochondria in heart failure: decrease in respirasomes and oxidative phosphorylation. Cardiovasc. Res. 80(1), 30-39 (2008). 
20. Dai DF, Johnson SC, Villarin JJ et al. Mitochondrial oxidative stress mediates angiotensin II-induced cardiac hypertrophy and Galphaq overexpression-induced heart failure. Circ. Res. 108(7), 837-846 (2011).

21. Fillmore N, Mori J, Lopaschuk GD. Mitochondrial fatty acid oxidation alterations in heart failure, ischaemic heart disease and diabetic cardiomyopathy. Brit. J. Pharmacol. 171(8), 2080-2090 (2014).

22. Nishida K, Otsu K. Autophagy during cardiac remodeling. J. Mol. Cell. Cardiol. 95, 11-18 (2016).

23. Lamb CA, Yoshimori T, Tooze SA. The autophagosome: origins unknown, biogenesis complex. Nat. Rev. Mol. Cell. Biol. 14(12), 759-774 (2013).

24. Kanamori H, Takemura G, Goto K et al. Autophagy limits acute myocardial infarction induced by permanent coronary artery occlusion. Am. J. Physiol. Heart. Circ. Physiol. 300(6), H2261-H2271 (2011).

25. Kanamori $\mathrm{H}$, Takemura G, Goto $\mathrm{K}$ et al. Resveratrol reverses remodeling in hearts with large, old myocardial infarctions through enhanced autophagy-activating AMP kinase pathway. Am. J. Pathol. 182(3), 701-713 (2013).

26. Sciarretta S, Volpe M, Sadoshima J. Mammalian target of rapamycin signaling in cardiac physiology and disease. Circ. Res. 114(3), 549-564 (2014).

27. Condorelli G, Roncarati R, Ross JJ et al. Heart-targeted overexpression of caspase3 in mice increases infarct size and depresses cardiac function. Proc. Natl Acad. Sci. USA 98(17), 9977-9982 (2001).

28. Teringova E, Tousek P. Apoptosis in ischemic heart disease. J. Transl. Med. 15(1), 87 (2017).

29. Liu Q. Lentivirus mediated interference of Caspase-3 expression ameliorates the heart function on rats with acute myocardial infarction. Eur. Rev. Med. Pharmacol. Sci. 18(13), 1852-1858 (2014).

30. Zhuo X, Wu Y, Ni Y et al. Isoproterenol instigates cardiomyocyte apoptosis and heart failure via AMPK inactivation-mediated endoplasmic reticulum stress. Apoptosis 18(7), 800-810 (2013).

31. Katsuki S, Matoba T, Koga J, Nakano K, Egashira K. Anti-inflammatory nanomedicine for cardiovascular disease. Front. Cardiovasc. Med. 4, 87 (2017).

32. Gheorghiade M, Albaghdadi M, Zannad F et al. Rationale and design of the multicentre, randomized, double-blind, placebo-controlled aliskiren trial on acute heart failure outcomes (ASTRONAUT). Eur. J. Heart Fail. 13(1), 100-106 (2011).

33. Dhillon S. Eplerenone: a review of its use in patients with chronic systolic heart failure and mild symptoms. Drugs 73(13), 1451-1462 (2013).

34. Xie M, Burchfield JS, Hill JA. Pathological ventricular remodeling: therapies: part 2 of 2. Circulation 128(9), 1021-1030 (2013).

35. Duan HY, Liu DM, Qian P et al. Effect of atorvastatin on plasma NT-proBNP and inflammatory cytokine expression in patients with heart failure. Genet. Mol. Res. 14(4), 15739-15748 (2015).

36. Mahalwar R, Khanna D. Pleiotropic antioxidant potential of rosuvastatin in preventing cardiovascular disorders. Eur. J. Pharmacol. 711(1-3), 57-62 (2013).

37. Prasad SK, Dargie HJ, Smith GC et al. Comparison of the dual receptor endothelin antagonist enrasentan with enalapril in asymptomatic left ventricular systolic dysfunction: a cardiovascular magnetic resonance study. Heart 92(6), 798-803 (2006).

38. Vecchis R, Cesaro A, Ariano C. Therapeutic benefits of phosphodiesterase-5 inhibition in chronic heart failure: a meta-analysis. Interv. Med. Appl. Sci. 9(3), 123-135 (2017).

39. Fraccarollo D, Widder JD, Galuppo $\mathrm{P}$ et al. Improvement in left ventricular remodeling by the endothelial nitric oxide synthase enhancer AVE9488 after experimental myocardial infarction. Circulation 118(8), 818-827 (2008).

40. Zhang J, Xue Y, Ni Y, Ning F, Shang L, Ma A. Size dependent effects of gold nanoparticles in ISO-induced hyperthyroid rats. Sci. Rep. 8(1), 10960 (2018).

41. Xiao N, Qi XY, Tang LN, Tan LL, Chen YQ, Zhao HM. VEGF promotes cardiac stem cells differentiation into vascular endothelial cells via the PI3K/Akt signaling pathway. Artif. Cells Nanomed. Biotechnol. 42(6), 400-405 (2014).

42. Jackson R, Tilokee EL, Latham $\mathrm{N}$ et al. Paracrine engineering of human cardiac stem cells with insulin-like growth factor 1 enhances myocardial repair. J. Am. Heart Assoc. 4(9), e2104 (2015).

43. Srivastava D, Ieda M, Fu J, Qian L. Cardiac repair with thymosin beta4 and cardiac reprogramming factors. Ann. NY Acad. Sci. 1270, 66-72 (2012).

44. Shrivastava S, Srivastava D, Olson EN, DiMaio JM, Bock-Marquette I. Thymosin beta4 and cardiac repair. Ann. NY Acad. Sci. 1194, 87-96 (2010).

45. Tillmanns J, Hoffmann D, Habbaba Y et al. Fibroblast activation protein alpha expression identifies activated fibroblasts after myocardial infarction. J. Mol. Cell. Cardiol. 87, 194-203 (2015).

46. Cerrada I, Ruiz-Sauri A, Carrero R et al. Hypoxia-inducible factor 1 alpha contributes to cardiac healing in mesenchymal stem cells-mediated cardiac repair. Stem. Cells Dev. 22(3), 501-511 (2013).

47. Duan J, Gherghe C, Liu D et al. Wnt1/betacatenin injury response activates the epicardium and cardiac fibroblasts to promote cardiac repair. EMBO J. 31(2), 429-442 (2012). 
48. Shinde AV, Frangogiannis NG. Fibroblasts in myocardial infarction: a role in inflammation and repair. J. Mol. Cell. Cardiol. 70, 74-82 (2014).

49. Higashi K, Yamada Y, Minatoguchi S et al. MicroRNA-145 repairs infarcted myocardium by accelerating cardiomyocyte autophagy. Am. J. Physiol. Heart Circ. Physiol. 309(11), H1813-H1826 (2015).

50. Yang MJ, Ku SH, Kim D et al. Enhanced cytoplasmic delivery of RAGE siRNA using bioreducible polyethylenimine-based nanocarriers for myocardial gene therapy. Macromol. Biosci. 15(12), 1755-1763 (2015).

51. Mekaru H, Lu J, Tamanoi F. Development of mesoporous silica-based nanoparticles with controlled release capability for cancer therapy. Adv. Drug Deliv. Rev. 95, 40-49 (2015).

52. Qiao Y, Zhu B, Tian A, Li Z. PEG-coated gold nanoparticles attenuate beta-adrenergic receptor-mediated cardiac hypertrophy. Int. J. Nanomedicine 12, 4709-4719 (2017).

53. Ravichandran R, Sridhar R, Venugopal JR, Sundarrajan S, Mukherjee S, Ramakrishna S. Gold nanoparticle-loaded hybrid nanofibers for cardiogenic differentiation of stem cells for infarcted myocardium regeneration. Macromol. Biosci. 14(4), 515-525 (2014).

54. Santos HA, Bimbo LM, Lehto VP, Airaksinen AJ, Salonen J, Hirvonen J. Multifunctional porous silicon for therapeutic drug delivery and imaging. Curr. Drug Discov. Technol. 8(3), 228-249 (2011).

55. Santos HA, Hirvonen J. Nanostructured porous silicon materials: potential candidates for improving drug delivery. Nanomedicine (Lond.) 7(9), 1281-1284 (2012).

56. Tölli MA, Ferreira MPA, Kinnunen SM et al. In vivo biocompatibility of porous silicon biomaterials for drug delivery to the heart. Biomaterials 35(29), 8394-8405 (2014).

57. Miragoli M, Ceriotti P, Iafisco M et al. Inhalation of peptide-loaded nanoparticles improves heart failure. Sci. Transl. Med. 10(424), n6205 (2018)

58. Lim GB. Heart failure: drug delivery using inhaled nanoparticles. Nat. Rev. Cardiol. 15(3), 133 (2018).

59. Roberts JR, McKinney W, Kan H et al. Pulmonary and cardiovascular responses of rats to inhalation of silver nanoparticles. J. Toxicol. Environ. Health A 76(11), 651-668 (2013).

60. Ivanov S, Dragoi AM, Wang X et al. A novel role for HMGB1 in TLR9-mediated inflammatory responses to CpG-DNA. Blood 110(6), 1970-1981 (2007).

61. Pandey RK, Prajapati VK. Molecular and immunological toxic effects of nanoparticles. Int. J. Biol. Macromol. 107, 1278-1293 (2018).

62. Bostan HB, Rezaee R, Valokala MG et al. Cardiotoxicity of nano-particles. Life Sci. 165, 91-99 (2016).

\section{- Summarizes cardiotoxity of various nanoparticles.}

63. Pattni BS, Chupin VV, Torchilin VP. New developments in liposomal drug delivery. Chem. Rev. 115(19), 10938-10966 (2015).

64. Jesorka A, Orwar O. Liposomes: technologies and analytical applications. Annu. Rev. Anal. Chem. (Palo Alto Calif.) 1, 801-832 (2008).

65. Mazur F, Bally M, Stadler B, Chandrawati R. Liposomes and lipid bilayers in biosensors. Adv. Colloid Interface Sci. 249, 88-99 (2017).

66. Kamaly N, Xiao Z, Valencia PM, Radovic-Moreno AF, Farokhzad OC. Targeted polymeric therapeutic nanoparticles: design, development and clinical translation. Chem. Soc. Rev. 41(7), 2971-3010 (2012).

67. Lassalle V, Ferreira ML. PLA nano- and microparticles for drug delivery: an overview of the methods of preparation. Macromol. Biosci. 7(6), 767-783 (2007).

68. Suarez S, Almutairi A, Christman KL. Micro- and nanoparticles for treating cardiovascular disease. Biomater. Sci. 3(4), 564-580 (2015).

69. Pagels RF, Prud'Homme RK. Polymeric nanoparticles and microparticles for the delivery of peptides, biologics, and soluble therapeutics. J. Control. Release 219, 519-535 (2015).

70. Dvir T, Bauer M, Schroeder A et al. Nanoparticles targeting the infarcted heart. Nano Lett. 11(10), 4411-4414 (2011).

71. Frangogiannis NG. Regulation of the inflammatory response in cardiac repair. Circ. Res. 110(1), 159-173 (2012).

72. Ranganathan A, Hindupur R, Vallikannan B. Biocompatible lutein-polymer-lipid nanocapsules: acute and subacute toxicity and bioavailability in mice. Mater. Sci. Eng. C Mater. Biol. Appl. 69, 1318-1327 (2016).

73. Yellon DM, Davidson SM. Exosomes: nanoparticles involved in cardioprotection? Circ. Res. 114(2), 325-332 (2014).

74. Mackie AR, Klyachko E, Thorne $\mathrm{T}$ et al. Sonic hedgehog-modified human $\mathrm{CD} 34^{+}$cells preserve cardiac function after acute myocardial infarction. Circ. Res. 111(3), 312-321 (2012).

75. Xiao J, Pan Y, Li XH et al. Cardiac progenitor cell-derived exosomes prevent cardiomyocytes apoptosis through exosomal miR-21 by targeting PDCD4. Cell. Death Dis. $7(6)$, e2277 (2016).

76. Wang C, Zhang C, Liu L et al. Macrophage-derived mir-155-containing exosomes suppress fibroblast proliferation and promote fibroblast inflammation during cardiac injury. Mol. Ther. 25(1), 192-204 (2017).

77. van Mil A, Vrijsen KR, Goumans M, Metz CH, Doevendans PA, Sluijter JP. MicroRNA-1 enhances the angiogenic differentiation of human cardiomyocyte progenitor cells. J. Mol. Med. (Berl.) 91(8), 1001-1012 (2013). 
78. Wang Y, Zhang L, Li Y et al. Exosomes/microvesicles from induced pluripotent stem cells deliver cardioprotective miRNAs and prevent cardiomyocyte apoptosis in the ischemic myocardium. Int. J. Cardiol. 192, 61-69 (2015).

79. Yu B, Kim HW, Gong M et al. Exosomes secreted from GATA-4 overexpressing mesenchymal stem cells serve as a reservoir of anti-apoptotic microRNAs for cardioprotection. Int. J. Cardiol. 182, 349-360 (2015).

80. Mocharla P, Briand S, Giannotti G et al. AngiomiR-126 expression and secretion from circulating CD34(+) and CD14(+) PBMCs: role for proangiogenic effects and alterations in type 2 diabetics. Blood 121(1), 226-236 (2013).

81. Feng Y, Huang W, Wani M, Yu X, Ashraf M. Ischemic preconditioning potentiates the protective effect of stem cells through secretion of exosomes by targeting Mecp2 via miR-22. PLoS ONE 9(2), e88685 (2014).

82. Katare R, Riu F, Mitchell K et al. Transplantation of human pericyte progenitor cells improves the repair of infarcted heart through activation of an angiogenic program involving micro-RNA-132. Circ. Res. 109(8), 894-906 (2011).

83. Deddens JC, Vrijsen KR, Girao H, Doevendans PA, Sluijter JP. Cardiac-released extracellular vesicles can activate endothelial cells. Ann. Transl. Med. 5(3), 64 (2017).

84. Hafiane A, Daskalopoulou SS. Extracellular vesicles characteristics and emerging roles in atherosclerotic cardiovascular disease. Metabolism 85, 213-222 (2018).

85. de Jong OG, Verhaar MC, Chen Y et al. Cellular stress conditions are reflected in the protein and RNA content of endothelial cell-derived exosomes. J. Extracell. Vesicle 1(1), 18396 (2012).

86. Fuhrmann G, Chandrawati R, Parmar PA et al. engineering extracellular vesicles with the tools of enzyme prodrug therapy. Adv. Mater. 30(15), e1706616 (2018).

87. Zhu Q, Heon M, Zhao Z, He M. Microfluidic engineering of exosomes: editing cellular messages for precision therapeutics. Lab. Chip 18(12), 1690-1703 (2018).

88. Vidiasheva IV, Abalymov AA, Kurochkin MA et al. Transfer of cells with uptaken nanocomposite, magnetite-nanoparticle functionalized capsules with electromagnetic tweezers. Biomater. Sci. 6(8), 2219-2229 (2018). 